\title{
THE NUMERICAL EVALUATION \\ OF A 2-D CAUCHY PRINCIPAL VALUE INTEGRAL ARISING IN BOUNDARY INTEGRAL EQUATION METHODS
}

\author{
GIOVANNI MONEGATO
}

Abstract. In this paper we consider the problem of computing 2-D Cauchy principal value integrals of the form

$$
f_{S} F\left(P_{0} ; P\right) d P, \quad P_{0} \in S
$$

where $S$ is either a rectangle or a triangle, and $F\left(P_{0} ; P\right)$ is integrable over $S$, except at the point $P_{0}$ where it has a second-order pole. Using polar coordinates, the integral is first reduced to the form

$$
\int_{\theta_{1}}^{\theta_{2}} f_{0}^{R(\theta)} \frac{f(r, \theta)}{r} d r d \theta
$$

where $f$ denotes the finite part of the (divergent) integral. Then ad hoc products of one-dimensional quadrature rules of Gaussian type are constructed, and corresponding convergence results derived. Some numerical tests are also presented.

\section{INTRODUCTION}

In several engineering problems, and in particular in the application of boundary element techniques to the solution of three-dimensional elasticity problems (see for example $[2,3,4,9,15,22]$ ), we have to deal with integrals of the form

$$
I\left(F ; P_{0}\right)=f_{S} F\left(P_{0} ; P\right) d P, \quad P_{0} \in S,
$$

where $S$ denotes either a rectangle or a triangle, and $F\left(P_{0} ; P\right)$ is integrable over $S$, except at the point $P_{0}$ where it has a second-order pole. These integrals are defined in a Cauchy principal value sense (see [20]), i.e.,

$$
I\left(F ; P_{0}\right)=\lim _{\varepsilon \rightarrow 0^{+}} \int_{S-C_{\varepsilon}} F\left(P_{0} ; P\right) d P, \quad P_{0} \in S,
$$

where $C_{\varepsilon}$ is a neighborhood of $P_{0}$ and $\varepsilon$ is the radius of the smallest circle containing $C_{\varepsilon}$.

Received by the editor July 8, 1991 and, in revised form, November 17, 1992 and March 17, 1993.

1991 Mathematics Subject Classification. Primary 65D32.

This work was supported by the Ministero dell' Università e della Ricerca Scientifica e Tecnologica of Italy. 
As we shall shortly see, the value of the limit in (1.2), whenever it exists, depends on the particular form of $C_{\varepsilon}$.

Following Tricomi's presentation (see [20]) of two-dimensional Cauchy principal value integrals, we introduce polar coordinates $(r, \theta)$ with the origin at $P_{0}$, and assume that in a neighborhood of $P_{0}$ we have

$$
F\left(P_{0} ; P\right)=\frac{f_{-2}\left(P_{0} ; \theta\right)}{r^{2}}+F_{1}\left(P_{0} ; P\right),
$$

where $r=\left|P-P_{0}\right|$ and $F_{1}\left(P_{0} ; P\right)$ is integrable over $S$. We can thus write

$$
\int_{S-C_{\varepsilon}} F\left(P_{0} ; P\right) d P=\int_{S-C_{\varepsilon}} F_{1}\left(P_{0} ; P\right) d P+\int_{0}^{2 \pi} f_{-2}\left(P_{0} ; \theta\right)\left[\int_{\delta(\varepsilon, \theta)}^{R(\theta)} \frac{d r}{r}\right] d \theta,
$$

where $R(\theta)$ and $\delta(\varepsilon, \theta)$ describe the contours of $S$ and $C_{\varepsilon}$, respectively. Taking the limit as $\varepsilon \rightarrow 0^{+}$, we obtain

$$
\begin{aligned}
\lim _{\varepsilon \rightarrow 0^{+}} & \int_{S-C_{\varepsilon}} F_{1}\left(P_{0} ; P\right) d P+\int_{0}^{2 \pi} f_{-2}\left(P_{0} ; \theta\right) \log R(\theta) d \theta \\
& -\lim _{\varepsilon \rightarrow 0^{+}} \int_{0}^{2 \pi} f_{-2}\left(P_{0} ; \theta\right) \log \delta(\varepsilon, \theta) d \theta .
\end{aligned}
$$

In particular, if we let $C_{\varepsilon}$ be a circle with center $P_{0}$ and radius $\varepsilon$, the last integral becomes

$$
\log \varepsilon \int_{0}^{2 \pi} f_{-2}\left(P_{0} ; \theta\right) d \theta
$$

and, as stated in [20], a necessary and sufficient condition for the existence of the limit (1.2) is

$$
\int_{0}^{2 \pi} f_{-2}\left(P_{0} ; \theta\right) d \theta=0
$$

If $C_{\varepsilon}$ is not a circle, but nevertheless $\delta(\varepsilon, \theta)$ is such that

$$
\lim _{\varepsilon \rightarrow 0^{+}} \frac{\delta(\varepsilon, \theta)}{\varepsilon}=\delta_{0}(\theta)
$$

then

$$
I\left(F ; P_{0}\right)=\int_{S} F_{1}\left(P_{0} ; P\right) d P+\int_{0}^{2 \pi} f_{-2}\left(P_{0} ; \theta\right) \log \frac{R(\theta)}{\delta_{0}(\theta)} d \theta .
$$

In the remaining part of the paper, $C_{\varepsilon}$ will denote the circle with center $P_{0}$ and radius $\varepsilon$, i.e., we shall set $\delta(\varepsilon, \theta)=\varepsilon$. In this case we have

$$
\lim _{\varepsilon \rightarrow 0^{+}} \int_{S-C_{\varepsilon}} \frac{f_{-2}\left(P_{0} ; \theta\right)}{r^{2}} d P=\int_{0}^{2 \pi} f_{0}^{R(\theta)} \frac{f_{-2}\left(P_{0} ; \theta\right)}{r} d r d \theta,
$$

where the symbol $f$ indicates the finite part (see (2.1) and (2.2) of [10]). If we use this latter definition, we need only consider a triangular region of integration with vertex at $P_{0}$, since any polygon $S$, with $P_{0} \in S$, can be thought of as the union of triangles, each one with the singularity at one vertex.

In $\S 3$ we will construct quadrature formulas for the more general case

$$
\int_{\theta_{1}}^{\theta_{2}} f_{0}^{R(\theta)} \frac{f(r, \theta)}{r} d r d \theta,
$$


where

$$
f_{0}^{R(\theta)} \frac{f(r, \theta)}{r} d r d \theta=\int_{0}^{R(\theta)} \frac{f(r, \theta)-f(0, \theta)}{r} d r+f(0, \theta) \log R(\theta) .
$$

Once we have a rule for this type of integral, it will then be straightforward to obtain a corresponding formula for our Cauchy principal value integral (1.1). Notice that (1.5) is always defined, while (1.1) exists if and only if (1.4) is satisfied. However, when this latter holds, (1.5) with $\theta_{1}=0, \theta_{2}=2 \pi$, and $f(r, \theta)=r^{2} F\left(P_{0} ; P\right)$ gives us the values of $(1.1)$.

Several papers have been written on the numerical evaluation of (1.1); see for instance $[5,8,18,19,21]$. However, the accuracy of the methods proposed in these papers appears to be poor, and the methods themselves are unsatisfactory also from a theoretical point of view. Also the algorithm recently presented in [6] has a drawback, since it requires some preliminary analytic computation.

The integration rules we propose for (1.5) are of product type; they are obtained by using a Gauss-Legendre (or Gauss-Lobatto) formula in the $\theta$ direction, and a Gauss-Radau (or Gauss-Lobatto)-type formula for the finitepart integral.

In $\S 2$ we present two very simple rules of Gaussian type for the finite-part integral and derive corresponding error estimates. In $\S 3$ we obtain a convergence result for the product formulas we propose to compute (1.5) and present some numerical tests.

\section{GAUSSIAN RULES FOR THE FINITE-PART INTEGRAL}

Here we examine two Gaussian rules for the numerical evaluation of a finitepart integral of the form

$$
f_{a}^{b} w(x) \frac{f(x)}{x-a} d x
$$

where $w(x)$ is the Jacobi weight function $w(x)=(b-x)^{\alpha}(x-a)^{\beta}, \alpha>-1$, $-1<\beta \leq 0$. The two rules (with $\alpha=\beta=0$ ) have already been suggested in [19], but without any analysis of their properties.

Although the case of importance for the approximation of (1.1) is $\alpha=\beta=$ 0 , here we will consider the more general integrals (2.1) since they may be of interest in their own. In deriving our results, we have to observe that the property

$$
f_{a}^{b} w(x) \frac{\lambda f(x)+\gamma g(x)}{x-a} d x=\lambda f_{a}^{b} w(x) \frac{f(x)}{x-a} d x+\gamma f_{a}^{b} w(x) \frac{g(x)}{x-a} d x
$$

is valid, whereas the inequality

$$
f_{a}^{b} w(x) \frac{|f(x) g(x)|}{x-a} d x \leq\|g\|_{\infty} f_{a}^{b} w(x) \frac{|f(x)|}{x-a} d x
$$

does not hold in general. Also, the usual linear change of variable rule is not 
permitted. For instance, we have

$$
\begin{array}{rl}
f_{a}^{b} & w(x) \frac{f(x)}{x-a} d x \\
\quad=\left(\frac{b-a}{2}\right)^{\alpha+\beta} f_{-1}^{1}(1-t)^{\alpha}(1+t)^{\beta} \frac{f\left(\frac{b-a}{2} t+\frac{b+a}{2}\right)}{t+1} d t \\
\quad+f(a)\left[f_{a}^{b} \frac{w(x)}{x-a} d x-\left(\frac{b-a}{2}\right)^{\alpha+\beta} f_{-1}^{1} \frac{(1-t)^{\alpha}(1+t)^{\beta}}{1+t} d t\right]
\end{array}
$$

(see (2.3) below).

The first Gaussian formula we propose is

$$
f_{a}^{b} w(x) \frac{f(x)}{x-a} d x=w_{0}^{\mathrm{I}} f(a)+\sum_{i=1}^{n} w_{i}^{\mathrm{I}} f\left(x_{i}^{I}\right)+R_{n}^{\mathrm{I}}(f),
$$

where the interior nodes $\left\{x_{i}^{\mathrm{I}}\right\}$ coincide with the images in $(a, b)$ of the zeros $-1<x_{1}^{(\alpha, \beta)}<x_{2}^{(\alpha, \beta)}<\cdots<x_{n}^{(\alpha, \beta)}<1$ of the $n$ th-degree Jacobi polynomial $P_{n}^{(\alpha, \beta)}(x)$ defined in $(-1,1)$; that is

$$
x_{i}^{\mathrm{I}}=\frac{b-a}{2} x_{i}^{(\alpha, \beta)}+\frac{b+a}{2}, \quad i=1, \ldots, n .
$$

The coefficients $\left\{w_{i}^{\mathrm{I}}\right\}$ are given by the expressions ${ }^{1}$

$$
\left\{\begin{array}{l}
w_{i}^{\mathrm{I}}=\left(\frac{b-a}{2}\right)^{\alpha+\beta} \frac{h_{i}^{(\alpha, \beta)}}{1+x_{i}^{(\alpha, \beta)}}, \quad i=1, \ldots, n \\
w_{0}^{\mathrm{I}}=f_{a}^{b} \frac{w(x)}{x-a} d x-\sum_{i=1}^{n} w_{i}^{\mathrm{I}}, \\
f_{a}^{b} \frac{w(x)}{x-a} d x=\left\{\begin{array}{r}
\frac{\log (b-a) \quad \text { if } \alpha=\beta=0,}{\frac{\alpha+\beta+1}{\beta}(b-a)^{\alpha+\beta} \frac{\Gamma(\alpha+1) \Gamma(\beta+1)}{\Gamma(\alpha+\beta+2)}} \\
\text { if } \alpha>-1,-1<\beta<0,
\end{array}\right.
\end{array}\right.
$$

where $\left\{h_{i}^{(\alpha, \beta)}\right\}$ denote the weights of the standard Gauss-Jacobi quadrature rule

$$
\int_{-1}^{1}(1-x)^{\alpha}(1+x)^{\beta} f(x) d x=\sum_{i=1}^{n} h_{i}^{(\alpha, \beta)} f\left(x_{i}^{(\alpha, \beta)}\right)+R_{n}^{G}(f),
$$

and $\Gamma(x)$ is the gamma function.

Formula (2.2) is of Radau type and can be defined as the rule we obtain when we replace $f(x)$ by the corresponding Lagrange interpolation polynomial.

Remark 1. Formula (2.2) can also be obtained by "subtracting out" first the singularity from (2.1), i.e., setting

$$
f_{a}^{b} w(x) \frac{f(x)}{x-a} d x=\int_{a}^{b} w(x) \frac{f(x)-f(a)}{x-a} d x+f(a) f_{a}^{b} \frac{w(x)}{x-a},
$$

${ }^{1}$ The expressions given in the third and fourth lines of (2.3) spring directly from the definition of finite part of an integral (see [7]). In the case $\alpha \neq 0, \beta=0$ there does not seem to exist a simple analytic expression for the corresponding integral, and thus one should compute it numerically. 
and then applying rule $(2.4)$ to the ordinary integral on the right-hand side. This implies, for instance, that the degree of precision of $(2.2)$ is $2 n$, i.e., $R_{n}^{\mathrm{I}}(f)=0$ whenever $f(x)$ is a polynomial of degree $\leq 2 n$. Furthermore, $R_{n}^{\mathrm{I}}(f)$ coincides with the remainder produced by the Gauss-Jacobi formula (2.4) when the latter is applied to the function $\frac{f(x)-f(a)}{x-a}$.

Lemma 1. For the coefficients $\left\{w_{i}^{\mathrm{I}}\right\}$ in (2.2) we have

$$
\sum_{i=0}^{n}\left|w_{i}^{\mathrm{I}}\right|= \begin{cases}O(\log n) & \text { if } \beta=0 \\ O\left(n^{-2 \beta}\right) & \text { if } \beta<0 .\end{cases}
$$

Proof. Since $w_{i}^{\mathrm{I}}>0, i=1, \ldots, n$, and

$$
\sum_{i=1}^{n} w_{i}^{\mathrm{I}}<\sum_{i=0}^{n}\left|w_{i}^{\mathrm{I}}\right|<\left|\int_{a}^{b} \frac{w(x)}{x-a} d x\right|+2 \sum_{i=1}^{n} w_{i}^{\mathrm{I}},
$$

it is sufficient to examine the behavior of the quantity

$$
\sum_{i=1}^{n} w_{i}^{\mathrm{I}}=\left(\frac{b-a}{2}\right)^{\alpha+\beta} \sum_{i=1}^{n} \frac{h_{i}^{(\alpha, \beta)}}{1+x_{i}^{(\alpha, \beta)}} .
$$

Notice that, given any $0<\delta<2$, we have

$$
\sum_{i=1}^{n} \frac{h_{i}^{(\alpha, \beta)}}{1+x_{i}^{(\alpha, \beta)}}=\sum_{\left|1+x_{i}^{(\alpha, \beta)}\right|<\delta} \frac{h_{i}^{(\alpha, \beta)}}{1+x_{i}^{(\alpha, \beta)}}+O(1) .
$$

Furthermore, since by $[17,(15.3 .14) \text { with } \alpha, \beta \text { interchanged, and (8.9.1) }]^{2}$ the relation

$$
h_{i}^{(\alpha, \beta)} \sim \theta_{i}^{2 \beta+1} n^{-1} \sim i^{2 \beta+1} n^{-2 \beta-2},
$$

where $\theta_{i}=\arccos x_{i}^{(\alpha, \beta)}$, holds uniformly with respect to $i$ and $n$ for $\varepsilon \leq$ $\theta_{i}<\pi$, we have

$$
\frac{h_{i}^{(\alpha, \beta)}}{1+x_{i}^{(\alpha, \beta)}} \sim \theta_{i}^{2 \beta-1} n^{-1} \sim i^{2 \beta-1} n^{-2 \beta} .
$$

When $\beta=0$, then

$$
\sum_{\left|1+x_{i}^{(\alpha, \beta)}\right|<\delta} w_{i}^{\mathrm{I}}<c \sum_{i=1}^{n} \frac{1}{i}=O(\log n),
$$

while for $\beta<0$,

$$
\sum_{\left|1+x_{i}^{(\alpha, \beta)}\right|<\delta} w_{i}^{\mathrm{I}}<c n^{-2 \beta} \sum_{i=1}^{n} \frac{1}{i^{1-2 \beta}}=O\left(n^{-2 \beta}\right) .
$$

Remark 2. We recall that the quantity $K_{n}=\sum_{i=0}^{n}\left|w_{i}^{\mathrm{I}}\right|$ represents the condition number for the quadrature sum. Its growth rate is very mild; it is the same as that for the case of a 1-D Cauchy principal value integral (see [13]) when we use interpolatory type rules based on zeros of Jacobi polynomials. Here are some

${ }^{2}$ And also recalling that $P_{n}^{(\alpha, \beta)}(x)=(-1)^{n} P_{n}^{(\beta, \alpha)}(-x)$. 
computed values in the case $a=-1, b=1, \alpha=\beta=0$ :

\begin{tabular}{c|ccccccc}
$n$ & 2 & 4 & 8 & 16 & 32 & 64 & 128 \\
\hline$K_{n}$ & 5.31 & 7.64 & 10.28 & 12.83 & 15.54 & 17.28 & 21.04
\end{tabular}

From Remark 1 it would be straightforward to derive for the remainder term $R_{n}^{\mathrm{I}}(f)$ the bound $O\left(n^{-k+1}\right) \omega\left(f^{(k)} ; n^{-1}\right)$, whenever $f \in C^{k}[a, b],, k \geq 1$. However, by proceeding differently, when $\beta=0$ we are able to obtain a better estimate (when $-1<\beta<0$, see Remark 3 below).

Theorem 1. Assume $f \in C^{k}[a, b], k \geq 1$, and $\beta=0$, in (2.2). Then

$$
R_{n}^{\mathrm{I}}(f)=O\left(n^{-k}\right) \omega\left(f^{(k)} ; n^{-1}\right),
$$

where $\omega\left(f^{(k)} ; \cdot\right)$ denotes the modulus of continuity of $f^{(k)}$ in $[a, b]$.

Proof. First we recall (see [16]) that for each integer $n \geq 2 k+1$ there exists a polynomial $q_{n}(x)$ of degree $n$ such that

$$
\left|f(x)-q_{n}(x)\right| \leq c\left(\frac{\sqrt{(b-x)(x-a)}}{n}\right)^{k} \omega\left(f^{(k)} ; n^{-1}\right) .
$$

Then (with $x_{0}^{\mathrm{I}}=a$ ) we write

$$
R_{n}^{\mathrm{I}}(f)=\int_{a}^{b} w(x) \frac{f(x)-q_{n}(x)}{x-a} d x-\sum_{i=0}^{n} w_{i}^{\mathrm{I}}\left[f\left(x_{i}^{\mathrm{I}}\right)-q_{n}\left(x_{i}^{\mathrm{I}}\right)\right],
$$

where now the integral is no longer defined in the finite-part sense, since $q_{n}(a)=$ $f(a)$ and $f \in C^{k}[a, b], k \geq 1$. Notice that, in view of (2.8), in the sum above we need only bound the quantity

$$
\sum_{i=1}^{n} w_{i}^{\mathrm{I}}\left(x_{i}^{\mathrm{I}}-a\right)^{k / 2} \leq c \sum_{i=1}^{n} \frac{h_{i}^{(\alpha, 0)}}{\sqrt{1+x_{i}^{(\alpha, 0)}}},
$$

since $x_{0}^{\mathrm{I}}-a=\frac{b-a}{2}\left(1+x_{i}^{(\alpha, 0)}\right)$ and $k \geq 1$. This task can be accomplished fairly easily once we recall the estimates used in (2.6). In this case, when $\varepsilon \leq \theta_{i}<\pi$ we obtain $h_{i}^{(\alpha, 0)}\left(1+x_{i}^{(\alpha, 0)}\right)^{-1 / 2} \sim n^{-1}$, and hence

$$
\sum_{i=1}^{n} \frac{h_{i}^{(\alpha, 0)}}{\sqrt{1+x_{i}^{(\alpha, 0)}}}=O(1) .
$$

The derivation of (2.7) is then straightforward.

The second Gaussian rule is of Lobatto type:

$$
f_{a}^{b} w(x) \frac{f(x)}{x-a} d x=w_{0}^{\mathrm{II}} f(a)+\sum_{i=1}^{n} w_{i}^{\mathrm{II}} f\left(x_{i}^{\mathrm{II}}\right)+w_{n+1}^{\mathrm{II}} f(b)+R_{n}^{\mathrm{II}}(f) .
$$

It is of interpolatory type and the nodes $\left\{x_{i}^{\mathrm{II}}\right\}$ are the images in $(a, b)$ of the zeros of the Jacobi polynomial $P_{n}^{(\alpha+1, \beta)}(x)$; its degree of precision is $2 n+1$. 
For the coefficients $w_{i}^{\mathrm{II}}$ it is not difficult to derive the following expressions:

$$
\left\{\begin{array}{l}
w_{i}^{\mathrm{II}}=\left(\frac{b-a}{2}\right)^{\alpha+\beta} \frac{\lambda_{i}^{(\alpha, \beta)}}{1+x_{i}^{(\alpha+1, \beta)}}, \quad i=1, \ldots, n, \\
w_{n+1}^{\mathrm{II}}=\frac{1}{2}\left(\frac{b-a}{2}\right)^{\alpha+\beta} \lambda_{n+1}^{(\alpha, \beta)}, \\
w_{0}^{\mathrm{II}}=f_{a}^{b} \frac{w(x)}{x-a} d x-\sum_{i=1}^{n+1} w_{i}^{\mathrm{II}},
\end{array}\right.
$$

where the $\lambda_{i}^{(\alpha, \beta)}$ 's are the weights of the Gauss-Radau formula

$$
\int_{-1}^{1} w(x) f(x) d x \approx \sum_{i=1}^{n} \lambda_{i}^{(\alpha, \beta)} f\left(x_{i}^{(\alpha+1, \beta)}\right)+\lambda_{n+1}^{(\alpha, \beta)} f(1) .
$$

Notice that rule (2.9) can also be obtained by applying (2.11) to the ordinary integral in (2.5).

The proof of the next lemma is very similar to that of Lemma 1.

Lemma 2. For the coefficients $\left\{w_{i}^{\mathrm{II}}\right\}$ in (2.8) we have

$$
\sum_{i=0}^{n+1}\left|w_{i}^{\mathrm{II}}\right|= \begin{cases}O(\log n) & \text { if } \beta=0, \\ O\left(n^{-2 \beta}\right) & \text { if } \beta<0 .\end{cases}
$$

Proof. It is sufficient to note that

$$
\lambda_{i}^{(\alpha, \beta)}=\frac{h_{i}^{(\alpha+1, \beta)}}{1-x_{i}^{(\alpha+1, \beta)}}, \quad i=1, \ldots, n,
$$

hence that

$$
\begin{aligned}
\sum_{i=1}^{n}\left|w_{i}^{\mathrm{II}}\right|= & \left(\frac{b-a}{2}\right)^{\alpha+\beta} \sum_{i=1}^{n} \frac{h_{i}^{(\alpha+1, \beta)}}{1-\left[x_{i}^{(\alpha+1, \beta)}\right]^{2}} \\
= & \frac{1}{2}\left(\frac{b-a}{2}\right)^{\alpha+\beta}\left[\sum_{\left|1+x_{i}^{(\alpha+1, \beta)}\right|<\delta} \frac{h_{i}^{(\alpha+1, \beta)}}{1+x_{i}^{(\alpha+1, \beta)}}+\sum_{\left|1-x_{i}^{(\alpha+1, \beta)}\right|<\delta} \frac{h_{i}^{(\alpha+1, \beta)}}{1-x_{i}^{(\alpha+1, \beta)}}\right] \\
& +O(1) .
\end{aligned}
$$

By the same argument used in the proof of Lemma 1 we then obtain that the last two sums are both $O(\log n)$ if $\beta=0$ and $O\left(n^{-2 \beta}\right)$ if $\beta<0$. Since $\lambda_{n+1}^{(\alpha, \beta)}=O(1)$ we have (2.12).

The next theorem is the analogue of Theorem 1 for rule (2.9) and can be proved in the same way.

Theorem 2. Assume $f(x) \in C^{k}[a, b], k \geq 1$, and $\beta=0$, in (2.9). Then

$$
R_{n}^{\mathrm{II}}(f)=O\left(n^{-k}\right) \omega\left(f^{(k)} ; n^{-1}\right) .
$$

Remark 3. Formulas (2.2) and (2.9) can be used to evaluate finite-part integrals of the form (see [7])

$$
f_{a}^{b} \frac{f(x)}{(x-a)^{1+\mu}} d x, \quad 0<\mu<1 .
$$


Indeed in such a situation it is sufficient to consider $w(x)=(x-a)^{-\mu}$, that is, $\alpha=0, \beta=-\mu$. When $f \in C^{1}[a, b]$, the rate of convergence of the formulas would be $O\left(n^{-1}\right) \omega\left(f^{(1)} ; n^{-1}\right)$ if $0<\mu<\frac{1}{2}, O\left(n^{-1} \log n\right) \omega\left(f^{(1)} ; n^{-1}\right)$ if $\mu=$ $\frac{1}{2}$, and $O\left(n^{-2+2 \mu}\right) \omega\left(f^{(1)} ; n^{-1}\right)$ if $\frac{1}{2}<\mu<1$. When $f \in C^{k}[a, b], k \geq 2$, the bound $O\left(n^{-k}\right) \omega\left(f^{(k)} ; n^{-1}\right)$ holds for any $0<\mu<1$.

\section{Product of Gaussian rules for the 2-D singular integral}

In this section we will use the quadrature formulas introduced in $\S 2$ to construct a product rule for the numerical evaluation of the integral

$$
I(f)=\int_{\theta_{1}}^{\theta_{2}} f_{0}^{R(\theta)} \frac{f(r, \theta)}{r} d r d \theta
$$

where the domain of integration is a triangle $T$ as in Figure 1 ; hence $R(\theta)=$ $\frac{d}{\sin \theta-c \cos \theta}$ if $t$ is represented by $y=c x+d$, or $R(\theta)=\frac{d}{\cos \theta}$ if $t$ is given by $x=d$.

The outer integral in (3.1) can be approximated by an $m$-point GaussLegendre rule, the inner one by (2.2) with $\alpha, \beta=0$. In so doing, we obtain

$$
I(f)=\frac{\theta_{2}-\theta_{1}}{2} \sum_{j=1}^{m} h_{j}^{(0,0)} \sum_{i=0}^{n} w_{i}^{\mathrm{I}} f\left(r_{i j}, \xi_{j}\right)+R_{m n}^{\mathrm{I}}(f),
$$

where

$$
\left\{\begin{array}{l}
r_{0 j}=0, \\
r_{i j}=\frac{R\left(\xi_{j}\right)}{2}\left(1+x_{i}^{(0,0)}\right), \quad i=1, \ldots, n, \\
w_{i}^{\mathrm{I}}=\frac{h_{j}^{(0,0)}}{1+x_{i}^{(0,0)}}, \quad i=1, \ldots, n, \\
w_{0}^{\mathrm{I}}=\log R\left(\xi_{j}\right)-\sum_{i=1}^{n} w_{i}^{\mathrm{I}}, \\
\xi_{j}=\frac{\theta_{2}-\theta_{1}}{2} x_{j}^{(0,0)}+\frac{\theta_{2}+\theta_{1}}{2}, \quad j=1, \ldots, m .
\end{array}\right.
$$

Denote by $H_{s}(\mu, \mu), 0<\mu \leq 1$, the space of functions $f(r, \theta)$ with all partial derivatives of order $j=0, \ldots, s$ continuous on a rectangular region $\mathfrak{R}=[0, R] \times\left[\theta_{1}, \theta_{2}\right], R=\max _{\theta_{1} \leq \theta \leq \theta_{2}}|R(\theta)|$, and such that each derivative of

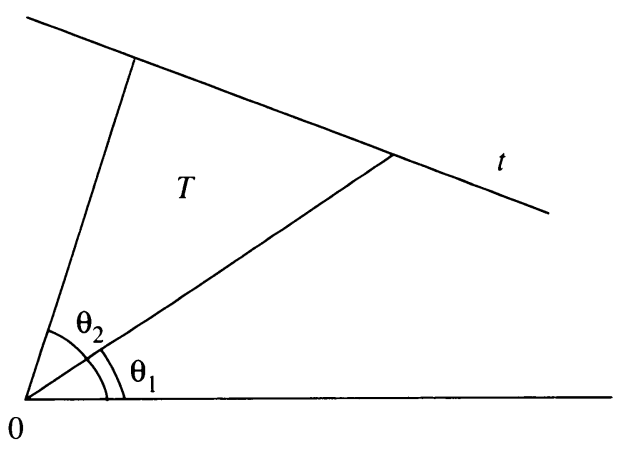

FIGURE 1 
order $s$ satisfies a Hölder condition of order $\mu$, i.e.,

$$
\left|f^{(s)}(\bar{r}, \bar{\theta})-f^{(s)}(r, \theta)\right| \leq c\left[|\bar{r}-r|^{\mu}+|\bar{\theta}-\theta|^{\mu}\right] .
$$

The rate of convergence of (3.2) is given by the following theorem.

Theorem 3. If in (3.2) we assume $f \in H_{s}(\mu, \mu), s \geq 1$, then we have

$$
R_{m n}^{\mathrm{I}}(f)=O\left(m^{1-s-\mu}+n^{1-s-\mu} \log n\right) .
$$

Proof. To obtain (3.3), we first write our integral (3.1) as follows:

$$
I(f)=\int_{\theta_{1}}^{\theta_{2}} F(\theta) d \theta, \quad F(\theta)=f_{0}^{R(\theta)} \frac{f(r, \theta)}{r} d r .
$$

We remark that in practical applications the function $F(\theta)$ is always analytic in $\left[\theta_{1}, \theta_{2}\right]$. For instance in the case $R(\theta)=\frac{d}{\sin \theta-c \cos \theta}$ it has infinitely many real poles at $\theta^{(k)}=\arctan (c) \pm k \pi, k=0,1,2, \ldots$, but they are all outside $\left[\theta_{1}, \theta_{2}\right]$ and very often sufficiently far away from it.

It is not difficult to show that when $f \in H_{s}(\mu, \mu)$ we have ${ }^{3} F \in H_{s}(\mu-\varepsilon)$, with $\varepsilon>0$ as small as we like (for a proof of this statement, see for example [12, Lemma 3]).

Applying the $m$-point Gauss-Legendre rule, we obtain

$$
I(f)=\frac{\theta_{2}-\theta_{1}}{2} \sum_{j=1}^{m} h_{j}^{(0,0)} F\left(\xi_{j}\right)+R_{m}^{G}(F) .
$$

Notice that since $F \in H_{s}(\mu-\varepsilon)$, the estimate $\left|R_{m}^{G}(F)\right|=\left|R_{m}^{G}\left(F-q_{m}\right)\right| \leq$ $2\left|\theta_{2}-\theta_{1}\right|\left\|F-q_{m}\right\|_{\infty}$, where $q_{m}$ denotes the $m$ th-degree polynomial of best uniform approximation, implies (see also [11]) $R_{m}^{G}(F)=O\left(m^{-s-\mu+\varepsilon}\right)$. Finally, we use (2.2) to approximate $F\left(\xi_{j}\right)$; we get

$$
I(f)=\frac{\theta_{2}-\theta_{1}}{2}\left[\sum_{j=1}^{m} h_{j}^{(0,0)} \sum_{i=0}^{n} w_{i}^{\mathrm{I}} f\left(r_{i j}, \xi_{j}\right)+\sum_{j=1}^{m} h_{j}^{(0,0)} R_{n}^{\mathrm{I}}\left(f ; \xi_{j}\right)\right]+R_{m}^{G}(F)
$$

hence

$$
R_{m n}^{\mathrm{I}}(f)=\frac{\theta_{2}-\theta_{1}}{2} \sum_{j=1}^{m} h_{j}^{(0,0)} R_{n}^{\mathrm{I}}\left(f ; \xi_{j}\right)+R_{m}^{G}(F) .
$$

Setting $f(r, \theta)=f(0, \theta)+r f_{0}(r, \theta)$, we let $p_{n, m}(r, \theta)$ be the polynomial of best (uniform) approximation of degree $n$ in $r$ and $m$ in $\theta$ for the function $f_{0}(r, \theta)$ defined on the rectangle $\mathfrak{R}$. Define

$$
\bar{p}_{n, m}(r, \theta)=f(0, \theta)+r p_{n, m}(r, \theta) .
$$

While in general $\bar{p}_{n, m}(r, \theta)$ is not a polynomial in the variable $\theta$, it is a polynomial of degree $n+1$ in the variable $r$. Thus, since (2.2) has degree of precision $2 n \geq n+1$, we can write

$$
\begin{aligned}
R_{n}^{\mathrm{I}}\left(f ; \xi_{j}\right)= & f_{0}^{R\left(\xi_{j}\right)} \frac{f\left(r, \xi_{j}\right)-\bar{p}_{n, m}\left(r, \xi_{j}\right)}{r} d r \\
& -\sum_{i=0}^{n} w_{i}^{\mathrm{I}}\left[f\left(r_{i j}, \xi_{j}\right)-\bar{p}_{n, m}\left(r_{i j}, \xi_{j}\right)\right]
\end{aligned}
$$

${ }^{3} H_{s}(\mu)$ denotes the space of functions $F$ whose derivatives of order up to $s$ exist and are continuous, and such that $F^{(s)}$ satisfies a Hölder condition of order $\mu$. 


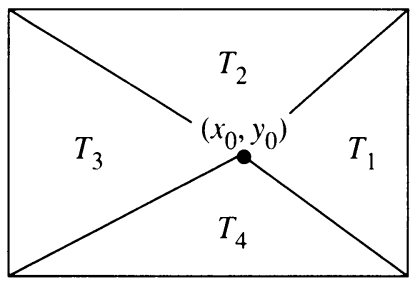

FIGURE 2

that is

$$
\begin{aligned}
R_{n}^{\mathrm{I}}\left(f ; \xi_{j}\right)= & \int_{0}^{R\left(\xi_{j}\right)}\left[f_{0}\left(r, \xi_{j}\right)-p_{n, m}\left(r, \xi_{j}\right)\right] d r \\
& -\sum_{i=0}^{n} w_{i}^{\mathrm{I}} r_{i j}\left[f_{0}\left(r_{i j}, \xi_{j}\right)-p_{n, m}\left(r_{i j}, \xi_{j}\right)\right] .
\end{aligned}
$$

After noticing that $f_{0} \in H_{s-1}(\mu, \mu)$, and recalling that (see $[11$, p. 90])

$$
\begin{gathered}
\left\|f_{0}(r, \theta)-p_{n, m}(r, \theta)\right\|_{\infty}=O\left(m^{1-s-\mu}+n^{1-s-\mu}\right), \\
\sum_{j=1}^{m} h_{j}^{(0,0)}=2 \text { and } \sum_{i=0}^{n}\left|w_{i}^{\mathrm{I}}\right|=O(\log n),
\end{gathered}
$$

from (3.6) and (3.5) we derive (3.3).

If the original region of integration is a polygon that has been subdivided into triangles, then it may be more efficient to use Lobatto-type rules. For instance, in the case of a single rectangle as in Figure 2, we suggest to use a Gauss-Lobatto rule for the integration of $F(\theta)$ in (3.4), and (2.2) for the integration along $r$ :

$$
I(f)=\sum_{j=0}^{m} \delta_{j} \sum_{i=0}^{n} w_{i}^{\mathrm{I}} f\left(r_{i j}, \eta_{j}\right)+R_{m n}^{\mathrm{II}}(f),
$$

where $\left\{\eta_{j}\right\}$ and $\left\{\delta_{j}\right\}$ denote the abscissae and the coefficients of the classical $m$-point Gauss-Lobatto formula associated with the interval $\left(\theta_{1}, \theta_{2}\right)$. Also, in this case we have a convergence rate like (3.3).

To test our formulas, we have applied them to the integrals

$$
\begin{gathered}
I_{1}\left(x_{0}, y_{0}\right)=f_{S} \frac{x-x_{0}}{\left[\left(x-x_{0}\right)^{2}+\left(y-y_{0}\right)^{2}\right]^{3 / 2}} d x d y \\
=\log \frac{\left[1-y_{0}+\sqrt{\left(1+x_{0}\right)^{2}+\left(1-y_{0}\right)^{2}}\right]\left[-1-y_{0}+\sqrt{\left(1-x_{0}\right)^{2}+\left(1+y_{0}\right)^{2}}\right]}{\left[-1-y_{0}+\sqrt{\left(1+x_{0}\right)^{2}+\left(1+y_{0}\right)^{2}}\right]\left[1-y_{0}+\sqrt{\left(1-x_{0}\right)^{2}+\left(1-y_{0}\right)^{2}}\right]}, \\
I_{2}\left(x_{0}, y_{0}\right)=f_{S} \frac{\left(x-x_{0}\right) e^{x}}{\left[\left(x-x_{0}\right)^{2}+\left(y-y_{0}\right)^{2}\right]^{3 / 2}} d x d y,
\end{gathered}
$$

where $S=[-1,1] \times[-1,1]$, and have obtained the results reported in Tables $1-3$.

The methods proposed in [18] and [19] are so inefficient mainly because they approximate the outer integral in $(3.1)$ by the trapezoidal rule over $(0,2 \pi)$. On this interval the function $R(\theta)$ has a first derivative which is discontinuous at the corners of the square. 
TABLE 1. Performance on $I_{1}$ with $\left(x_{0}, y_{0}\right)=(.3606231751$, $.3606231751)(1)$ and $\left(x_{0}, y_{0}\right)=(.5479477112, .9509446082)(2)$

\begin{tabular}{rrrcc}
\hline & \multicolumn{3}{c}{ rel. error } \\
& $n$ & \# points & $(1)$ & $(2)$ \\
\hline & \multicolumn{3}{c}{ rule $(3.7)$} \\
3 & 1 & 16 & $4.37 E-2$ & $3.49 E-1$ \\
4 & 1 & 24 & $2.03 E-3$ & $1.42 E-1$ \\
5 & 1 & 32 & $2.53 E-4$ & $6.47 E-2$ \\
6 & 1 & 40 & $3.06 E-5$ & $3.23 E-2$ \\
7 & 1 & 48 & $3.90 E-6$ & $1.70 E-2$ \\
8 & 1 & 56 & $5.14 E-7$ & $9.24 E-3$ \\
9 & 1 & 64 & $6.95 E-8$ & $5.17 E-3$ \\
10 & 1 & 72 & $9.57 E-9$ & $2.95 E-3$ \\
16 & 1 & 120 & $8.21 E-14$ & $1.31 E-4$ \\
32 & 1 & 248 & - & $7.65 E-8$ \\
& \multicolumn{3}{c}{ method $[19]$} \\
8 & 8 & 64 & $2.85 E-1$ & 4 \\
32 & 32 & 1024 & 4 & $3.02 E-3$ \\
\hline
\end{tabular}

TABLE 2. Performance on $I_{1}$ with $\left(x_{0}, y_{0}\right)=(.4, .1)(1),\left(x_{0}, y_{0}\right)$ $=(.6, .2)(2)$, and $\left(x_{0}, y_{0}\right)=(.8, .4)(3)$.

\begin{tabular}{rrrrrr}
\hline$m$ & $n$ & \# points & $(1)$ & $(2)$ & $(3)$ \\
\hline & & rule $(3.7)$ & & \\
3 & 1 & 16 & $4.66 E-2$ & $6.27 E-2$ & $8.66 E-2$ \\
5 & 1 & 32 & $8.46 E-5$ & $1.88 E-4$ & $6.10 E-4$ \\
7 & 1 & 48 & $6.09 E-7$ & $3.06 E-6$ & $2.81 E-5$ \\
9 & 1 & 64 & $5.82 E-9$ & $7.50 E-8$ & $2.04 E-6$ \\
12 & 1 & 88 & $7.53 E-12$ & $4.29 E-10$ & $5.98 E-8$ \\
& & & method $[18]$ & & \\
18 & 20 & 360 & $1.12 E-3$ & $1.19 E-3$ & $3.61 E-4$ \\
36 & 20 & 720 & $1.07 E-3$ & $1.53 E-4$ & $1.80 E-4$ \\
72 & 20 & 1440 & $3.61 E-4$ & $1.10 E-5$ & $6.70 E-6$ \\
\hline
\end{tabular}

TABle 3. Performance of rule (3.7) on $I_{2}$

\begin{tabular}{rlcc}
\hline$m$ & $n$ & \# points & rel. error \\
\hline & \multicolumn{2}{c}{$I_{2}(.5, .5)=2.04712179371331^{5}$} \\
8 & 4 & 140 & $3.43 E-8$ \\
16 & 4 & 300 & $1.10 E-9$ \\
16 & 8 & 540 & $7.03 E-13$ \\
& $I_{2}(.9, .9)=-4.78691846480268^{5}$ \\
8 & 4 & 140 & $3.23 E-4$ \\
16 & 4 & 300 & $8.97 E-9$ \\
32 & 8 & 1116 & $1.20 E-11$ \\
\hline
\end{tabular}

4 Value not reported in [19].

5 An explicit formula for $I_{2}$ is not available; however, all 15 decimal digits obtained by using our rule with $m=32, n=8$ in case (1), and $m=64, n=8$ in case (2), are believed to be correct since they coincide with those obtained with $m=n=64$ and $m=n=128$, respectively. 
All computations have been performed on a personal computer using 16-digit arithmetic.

\section{ACKNOWLEDGMENT}

The author is grateful to the referees for their careful reading and useful comments.

\section{BIBLIOGRAPHY}

1. C. A. Brebbia, J. C. F. Telles, and L. C. Wrobel, Boundary element techniques, SpringerVerlag, Berlin, 1984.

2. T. A. Cruse, Numerical solutions in three-dimensional elastostatics, Internat. J. Solids and Structures 5 (1969), 1259-1274.

3. ysis, Comput. \& Structures 3 (1973), 509-527.

4. T. A. Cruse and R. B. Wilson, Advanced applications of boundary-integral equation methods, Nuclear Engrg. Des. 46 (1978), 223-234.

5. B. G. Gabdulkhaev and L. A. Onezov, Cubature formulas for singular integrals, Izv. Vyssh. Uchebn. Zaved. Mat. 7 (1976), 100-105; English transl. in Soviet Math. (Iz. VUZ).

6. M. Guiggiani and A. Gigante, A general algorithm for multidimensional Cauchy principal value integrals in the boundary element method, ASME J. Appl. Mech. 57 (1990), 907-915.

7. J. Hadamard, Lectures on Cauchy's problem in linear partial differential equations, Yale Univ. Press, 1923; Dover Publ., 1952 .

8. J. G. Kazantzakis and P. S. Theocaris, The evaluation of certain two-dimensional singular integrals used in three-dimensional elasticity, Internat. J. Solids and Structures 15 (1979), 203-207.

9. G. Krishnasamy, L. W. Schmerr, T. J. Rudolphi, and F. J. Rizzo, Hypersingular boundary integral equations: some applications in acoustic and elastic scattering, ASME J. Appl. Mech. 57 (1990), 404-414.

10. H. R. Kutt, The numerical evaluation of principal value integrals by finite-part integration, Numer. Math. 24 (1975), 205-210.

11. G. G. Lorentz, Approximation of functions, Holt, Rinehart and Winston, New York, 1966.

12. G. Monegato, Convergence of product formulas for the numerical evaluation of certain two-dimensional Cauchy principal value integrals, Numer. Math. 43 (1984), 161-173.

13. __ On the weights of certain quadratures for the numerical evaluation of Cauchy principal value integrals and their derivatives, Numer. Math. 50 (1987), 273-281.

14. A. G. Ramm and A. van der Sluis, Calculating singular integrals as an ill-posed problem, Numer. Math. 57 (1990), 139-145.

15. F. J. Rizzo and D. J. Shippy, An advanced boundary integral equation method for threedimensional thermoelasticity, Internat. J. Numer. Methods Engrg. 11 (1977), 1753-1768.

16. P. O. Runck, Bemerkungen zu den Approximationssätzen von Jackson und Jackson-Timan, Abstrakte Räume und Approximation (P. L. Butzer, B. Szökefalvi-Nagy, eds.), ISNM, vol. 10, Birkhäuser Verlag, Basel, 1969, pp. 303-308.

17. G. Szegö, Orthogonal polynomials, Amer. Math. Soc. Colloq. Publ., vol. 23, Amer. Math. Soc., Providence, RI, 1975.

18. P. S. Theocaris, N. I. Ioakimidis, and J. G. Kazantzakis, On the numerical evaluation of two-dimensional principal value integrals, Internat. J. Numer. Methods Engrg. 14 (1980), 629-634. 
19. P. S. Theocaris, Modified Gauss-Legendre, Lobatto and Radau cubature formulas for the numerical evaluation of 2-D singular integrals, Internat. J. Math. Math. Sci. 6 (1983), 567-587.

20. F. Tricomi, Equazioni integrali contenenti il valor principale di un integrale doppio, Math. Z. 27 (1928), 87-133.

21. G. Tsamasphyros and P. S. Theocaris, Cubature formulas for the evaluation of surface singular integrals, BIT 19 (1979), 368-377.

22. J. Weaver, Three-dimensional crack analysis, Internat. J. Solids and Structures 13 (1977), 321-330.

Dipartimento di Matematica, Politecnico di Torino, Torino, Italia

E-mail address: monegato@itopoli.bitnet 